\title{
A Feasible Methodological Framework for Uncertainty Analysis and Diagnosis of Atmospheric Chemical Transport Models
}

Zhijiong Huang ${ }^{\mathrm{a}}$, Junyu Zheng ${ }^{\mathrm{a}, *}$, Jiamin $\mathrm{Ou}^{\mathrm{c}}$, Zhuangmin Zhong ${ }^{\mathrm{a}}$, Yuqi $\mathrm{Wu}^{\mathrm{b}}$, Min Shao ${ }^{\mathrm{a}}$

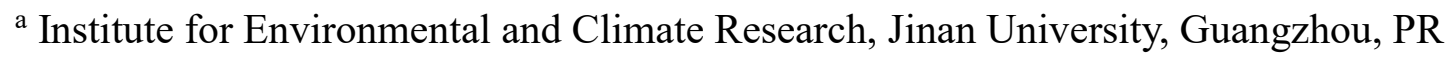
China

${ }^{\mathrm{b}}$ School of Environment and Energy, South China University of Technology, Guangzhou, PR China

${ }^{\mathrm{c}}$ School of International Development, University of East Anglia, Norwich NR4 7JT, UK

KEYWORDS: Uncertainty analysis, HDDM, SRSM, model diagnosis 


\section{ABSTRACT}

The current state of quantifying uncertainty in chemical transport models (CTM) is often limited and insufficient due to numerous uncertainty sources and inefficient or inaccurate uncertainty propagation methods. In this study, we proposed a feasible methodological framework for CTM uncertainty analysis, featuring sensitivity analysis to filter important model inputs and a new reduced-form model (RFM) that couples the High-order Decoupled Direct Method (HDDM) and the Stochastic Response Surface Model (SRSM) to boost uncertainty propagation. Compared with the SRSM, the new RFM approach is $64 \%$ more computationally efficient while maintaining high accuracy. The framework was applied to $\mathrm{PM}_{2.5}$ simulations in the Pearl River Delta (PRD) region, and identified five precursor emissions, two species in lateral boundary conditions (LBCs) and three meteorological inputs out of 203 model inputs as important model inputs based on sensitivity analysis. Among these selected inputs, primary $\mathrm{PM}_{2.5}$ emissions, $\mathrm{PM}_{2.5}$ concentrations of LBCs and wind speed were key uncertainty sources, which collectively contributed $81.4 \%$ to the total uncertainty in $\mathrm{PM}_{2.5}$ simulations. Also, when evaluated against observations, we found that there were systematic underestimates in $\mathrm{PM}_{2.5}$ simulations, which can be attributed to the twoproduct method that describes the formation of secondary organic aerosol. 
24

25
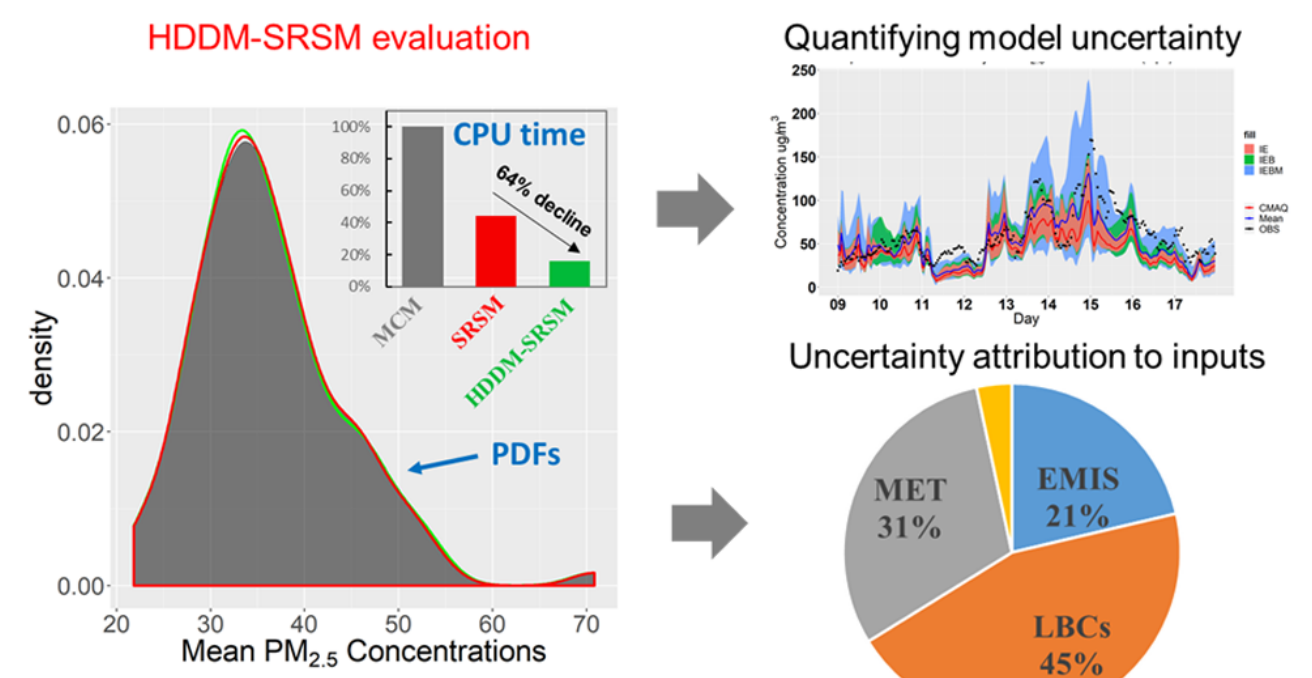

26

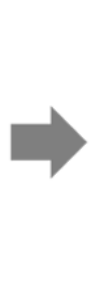

Uncertainty attribution to inputs

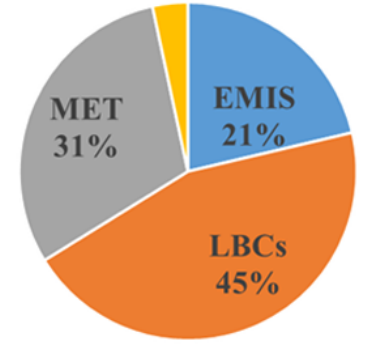




\section{INTRODUCTION}

Atmospheric chemical transport models (CTMs) are critical tools for regulatory decision making, attainment demonstration, and air quality forecasting ${ }^{1,2}$. However, current CTMs still have substantial bias in simulating air pollutant concentrations, particularly in reproducing $\mathrm{PM}_{2.5}$ concentrations and their species compared against observations ${ }^{3}$. Various sources of uncertainty exist in developing and applying CTMs models, including the parametric uncertainty associated with input data or parameters and the structural uncertainty arising from simplifications of complex chemical and physical processes ${ }^{4}$. Uncertainty analysis is an effective mean to improve model performance by identifying and diagnosing key sources of uncertainty ${ }^{2,5-7}$. Although some attempts have been made to characterize uncertainties of atmospheric models in recent decades, ${ }^{2,8}$ better quantification of uncertainties in CTMs remains a top research priority for atmospheric scientists ${ }^{9,10}$.

Traditional approaches for uncertainty analysis of CTMs are computationally expensive, particularly for traditional Monte Carlo method (MCM) $)^{11,12}$ or Latin Hypercube Sampling (LHS) ${ }^{13}$ to propagate uncertainties. Some approaches have been proposed to address this limitation, featuring the use of reduced-form models (RFM) including: Stochastic Response Surface Model $(\mathrm{SRSM})^{1}$ and Probabilistic Collocation Method (PCM) $)^{14-17}$ based on the polymonial chaos expansions (PCEs), the reduced-form model based on High-order Decoupled Direct Method (RFM-HDDM) $)^{6,8,18}$ and the recently developed stepwise-based HDDM (SB-HDDM) ${ }^{7}$. These approaches all use an polynomial expansion instead of the original CTM to propagate uncertainties. However, the RFMHDDM has significant biases in predicting nonlinear responses when there are high uncertainties in model inputs ${ }^{7}{ }^{19}$. The SB-HDDM partly 
overcomes this limitation but still has biases because it ignores the highorder cross sensitivities and assumes that the interaction among inputs is linear. The SRSM and PCM can help improve the accuracy of propagating uncertainties, but its efficiency dramatically decreases with the increase of uncertainty sources, which limits its application to CTMs that have numerous uncertainty sources ${ }^{16,20,21}$.

As the scientific understanding of atmospheric physical and chemical processes evolves, CTMs will become more comprehensive with more model inputs and detailed model structures ${ }^{22,23}$. This will most likely bring greater challenge in conducting uncertainty analysis since it requires more data collection to quantify additional uncertainty sources and more computational cost to propagate them, even if RFM approaches are used. Therefore, in order to make it possible to conduct uncertainty analysis of CTMs, two critical issues must be addressed: how to ensure the accuracy of propagating uncertainties and how to improve the efficiency when there are many uncertainty sources.

In this study, we proposed a feasible methodological framework to quantify uncertainties of CTMs. The framework uses a sensitivity analysis to filter out unimportant model inputs and make it feasible to apply RFM approaches for efficient uncertainty propagation. Additionally, it incorporates a novel approach to improve the accuracy and efficiency of uncertainty propagation. We applied the framework to a case study of the uncertainty analysis of $\mathrm{PM}_{2.5}$ modeling in the Pearl River Delta (PRD) using the CMAQv5.0.2, a widely used chemical transport model, to demonstrate its feasibility in model uncertainty analysis, and how uncertainty analysis can help model diagnosis. 


\section{MATERIALS AND METHODS}

\subsection{The methodological framework for efficient uncertainty} analysis of CTMs.

Previous studies have explored uncertainty analysis of CTMs, but there is still no consistent integrated methodological framework to quantify uncertainty. Here, we proposed a conceptual methodological framework to help guide the uncertainty analysis of CTMs (Figure 1). The framework involves 6 steps: the use of (1) sensitivity analysis and (2) estimation of input uncertainties to select important model inputs for further uncertainty analysis, (3) propagation of uncertainty through models using a RFM approach to obtain output uncertainties, (4) quantification of model output uncertainties, (5) evaluation of output uncertainties with observations, and (6) identification of key uncertainty sources to guide model improvements.

The purpose of sensitivity analysis is to filter out insensitive inputs to reduce the number of uncertainty sources for further uncertainty analysis. This is reasonable because most of the key uncertainty sources are sensitive, particularly in cases that all input uncertainties are approximately of the same magnitude ${ }^{24}$. In a few cases, an insensitive input may also be a key uncertainty source if its uncertainty is extremely large. Therefore, estimating input uncertainties is recommended to assist in selecting important inputs for further uncertainty analysis. The sensitivity of a model input is quantified using the relative sensitivity coefficient (RSC), defined as the ratio of the absolute value of the first-order sensitivity coefficient to the base-level concentration. The HDDM and the Brute-Force Method $(\mathrm{BFM})^{25}$ are two commonly applied approaches to calculate sensitivity coefficients of CTMs. In this study, sensitivity coefficients of emission rates, lateral boundary conditions (LBCs), and chemical reaction rates were calculated using the HDDM. For other inputs that are not available in the HDDM, e.g., meteorological fields, the BFM was used. 
RFM approaches are generally adopted to propagate uncertainties of CTMs due to their high efficiency. Current RFM approaches still have limitations with regards to inaccuracy and/or inefficiency. Here, we developed a new RFM approach by coupling the SRSM with HDDM. This approach can improve efficiency while maintaining accuracy in propagating uncertainty of CTMs (see Section 2.2 for more details).

Comparing uncertainty with observations can evaluate whether uncertainties in CTMs are reasonably quantified in terms of the spread and probabilistic prediction. Here, we integrated several methods based on previous assessments of ensemble simulations ${ }^{18,26}$ to evaluate the output uncertainty performance. The Fractional Error (FE) and Fractional Bias $(\mathrm{FB})^{27}$ measured the superiority of the mean of uncertainty to a single simulation. The Probability Integral Transform (PIT) ${ }^{28}$ was used to measure the spread-skill relationship between uncertainty and simulation error. The Reliability Diagram $(\mathrm{RD})^{29}$ quantified the reliability and resolution of a probabilistic forecast. Details of the evaluation are summarized in S3 of SI.

Identifying the key sources of uncertainty provides guidance for future model improvement. Here, we used a variance-based method proposed by Huang et al. ${ }^{7}$ to assign model output uncertainties to uncertainty sources. The contribution is calculated as the ratio of the variance of model outputs induced by a single uncertainty source to the total variance of model outputs induced by all uncertainty sources (S4 of SI).

Steps 2 and 4 are performed using statistical approaches and more details are available in S2 of Supporting Information (SI). 


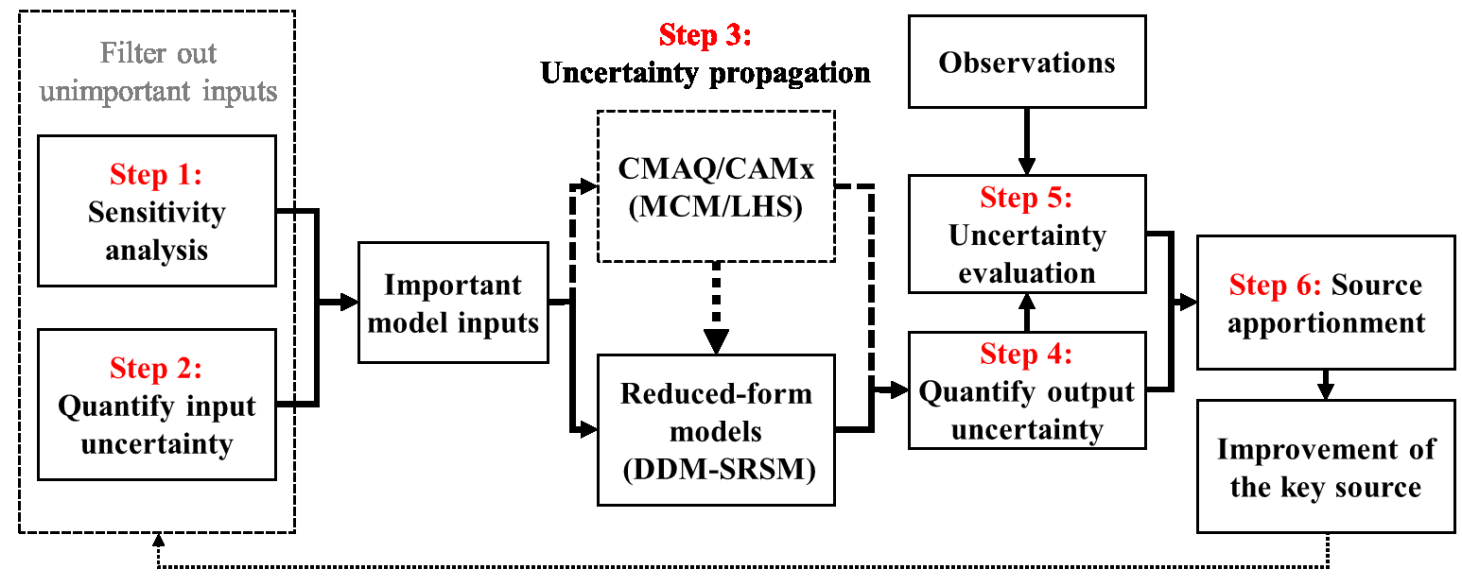

Figure 1. The framework of efficient uncertainty analysis for CTMs

\subsection{A novel RFM-based uncertainty propagation approach: HDDM-SRSM}

The PCE-based approach (SRSM and PCM) is an efficient mean for uncertainty propagation; however, its efficiency decreases rapidly as its upfront model runs grow with the increase of uncertainty sources ${ }^{16}$. Isukapalli et al. ${ }^{20}$ showed that coupling the SRSM with sensitivity information can reduce the number of up-front model runs. Based on this, we developed a more efficient uncertainty propagation method, HDDM-SRSM, by coupling the SRSM with sensitivity coefficients calculated by HDDM (Figure 2).

Here, we briefly described the four steps for approximating CTM using the $M$-order HDDM-SRSM (see S5 of SI for complete details). First, the input uncertainty is transformed into a standard random variable (SRV) to facilitate a consistent representation of the model inputs and outputs as functions of mathematically tractable random variables. Second, the model output is expressed as a PCE based on multidimensional Hermite polynomials with $N$ unknown coefficients (eq 1). The maximum order of Hermite polynomials is $M$. These two steps mainly follow the methodology of the SRSM and PCM ${ }^{14-17}$.

Collocation points that correspond to the roots of the Hermite 
polynomial of one degree higher than the order of the PCE were previously used to obtain unknown coefficients. Typically, $N$ collocation points (one collocation point requires one model run) were required to form $N$ equations based on eq. 1 to solve $N$ unknown coefficients ${ }^{14}$. However, in the HDDM-SRSM, the first-order sensitivity coefficients calculated by HDDM in each model run could also form equations according to eq. 2. Thus, eq. 1 in conjunction with sensitivity coefficients could greatly decrease the required number of collocation points, but also in turn enhances the dependence of the PCE on the choice of collocation points. To reduce the dependence and obtain a robust PCE, we used the regression method $^{20}$, which recommends twice as the least required number of collocation points, to estimate unknown coefficients. The number of model runs $N_{\text {run }}$ in HDDM-SRSM depends on the number of inputs $(m)$, sensitivity coefficients $(k)$, and the order of Hermite polynomials (M) (eq. 4).

Fourth, the probability distribution function (PDF) derived from the $M$-order HDDM-SRSM is compared with MCM-derived PDF to evaluate the accuracy of approximation. If the two PDFs agree, the approximation based on the $M$-order HDDM-SRSM is used for uncertainty propagation. Otherwise, a higher-order HDDM-SRSM is used and the four aforementioned steps are repeated.

$$
y=a_{0}+\sum_{i_{1}=1}^{n} a_{i_{1}} \Gamma_{1}\left(\xi_{i_{1}}\right)+\sum_{i_{1}=1}^{n} \sum_{i_{2}=1}^{i_{1}} a_{i_{1} i_{2}} \Gamma_{2}\left(\xi_{i_{1}}, \xi_{i_{2}}\right)+\sum_{i_{1}=1}^{n} \sum_{i_{2}=1}^{i_{1}} \sum_{i_{3}=1}^{i_{2}} a_{i_{1} i_{2} i_{3}} \Gamma_{3}\left(\xi_{i_{1}}, \xi_{i_{2}}, \xi_{i_{3}}\right)+\cdots
$$

$$
\frac{\partial y}{\partial \xi_{i_{1}}}=a_{i_{1}} \frac{\partial \Gamma_{1}\left(\xi_{i_{1}}\right)}{\partial \xi_{i_{1}}}+\sum_{i_{2}=1}^{n} a_{i_{1} i_{2}} \frac{\partial \Gamma_{2}\left(\xi_{i_{1}}, \xi_{i_{2}}\right)}{\partial \xi_{i_{1}}}+\sum_{i_{2}=1}^{n} \sum_{i_{3}=1}^{i_{2}} a_{i_{1} i_{2} i_{3}} \frac{\partial \Gamma_{3}\left(\xi_{i_{1}}, \xi_{i_{2}}, \xi_{i_{3}}\right)}{\partial \xi_{i_{1}}}
$$

$$
\Gamma_{\mathrm{m}}\left(\xi_{i_{1}}, \ldots, \xi_{i_{m}}\right)=(-1)^{m} e^{0.5\{\xi\}\{\xi\}^{T}} \frac{\partial m}{\partial \xi_{i_{1}, \ldots}, \xi_{i_{m}}} e^{-0.5\{\xi\}\{\xi\}^{T}}
$$

$$
N_{\text {run }}=\text { Floor }\left(\frac{2 \times(\mathrm{m}+\mathrm{n}) !}{\mathrm{m} ! \mathrm{n} ! \times k}\right)
$$

where $y$ is the model output; $a_{i_{1}}, a_{i_{1} i_{2}}$, and $a_{i_{1} i_{2} i_{3}}$ are unknown coefficients to be estimated; and $\Gamma_{m}\left(\xi_{i_{1}}, \ldots, \xi_{i_{m}}\right)$ are multidimensional 
Hermite polynomials of order $m$. The accuracy of approximation increases with the order of Hermite polynomials. In general, a second-order polynomial is recommended as a first attempt. $\xi_{i}$ is the SRV of model input $i$ and $n$ represents the number of inputs. $\frac{\partial y}{\partial \xi_{i_{1}}}$ denotes the first-order sensitivity coefficient to model input $i_{1}$.

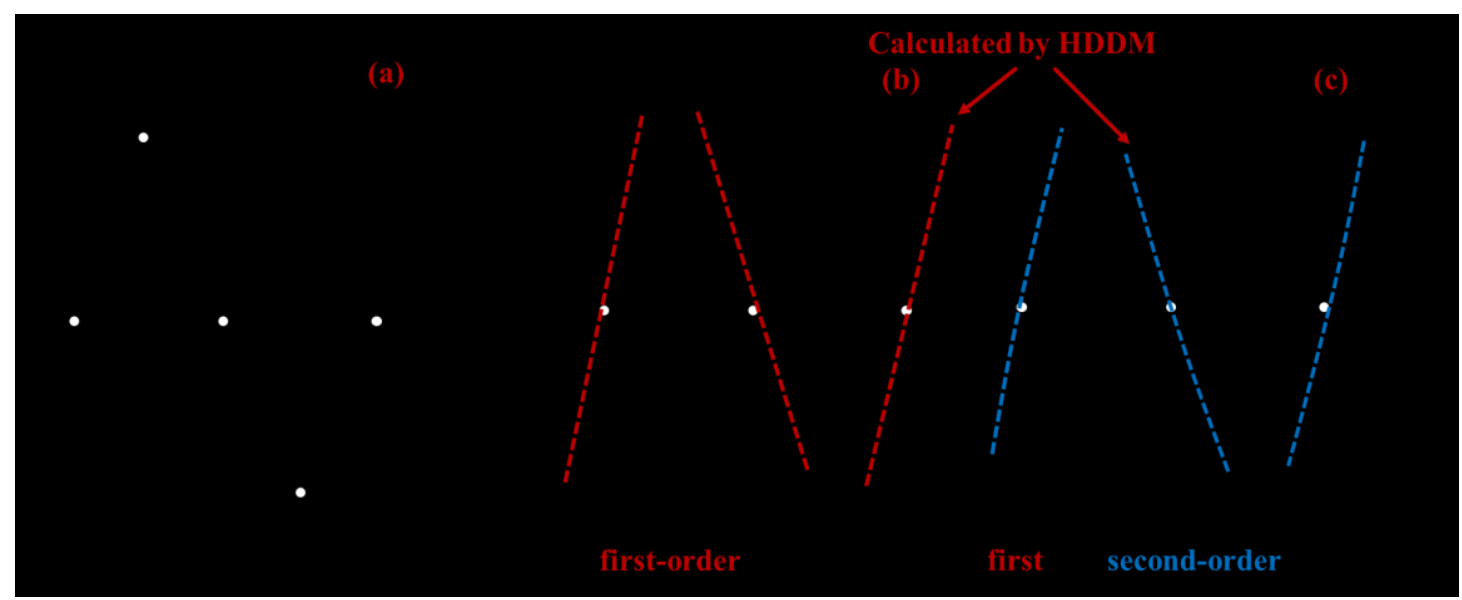

Figure 2. A depiction of the HDDM-SRSM method. (a) The SRSM method requires at least five well-distributed model runs to accurately approximate the model response. (b) Since sensitivity coefficients can constrain the shape of model responses, coupling first-order sensitivity coefficients with concentrations can reduce the number of model runs required for approximation. Here, only three model runs are needed to obtain a similar approximation. (c) Adding second-order sensitivity coefficients can further improve the accuracy of approximation, but it might not reduce the number of model runs due to overfitting (Figure S1).

\subsection{A case study}

We applied the framework to analyze and diagnose uncertainties in $\mathrm{PM}_{2.5}$ simulations in the PRD region with the use of the CMAQv5.0.2 model coupled with the WRF model. The detailed configuration for these two models are shown in S6 of SI. Because we did not intend to evaluate how model mechanisms or parameterization schemes impact model outputs, all uncertainty sources considered in this study are parametric. These sources included emissions of $\mathrm{NOx}, \mathrm{SO}_{2}, \mathrm{VOCs}, \mathrm{PM}_{2.5}$ and $\mathrm{NH}_{3}$; concentrations of $\mathrm{PM}_{2.5}, \mathrm{O}_{3}, \mathrm{HNO}_{3}, \mathrm{SO}_{2}, \mathrm{NOx}$, and $\mathrm{NH}_{3}$ in LBCs; 11 
meteorological fields provided by the Meteorology-Chemistry Interface Processor (MCIP) and 182 chemical reaction rates in CB05. Meteorological uncertainties for relative humidity, cloud cover, inverse of Monin-Obukhov length (MOLI), planetary boundary layer height (PBL), pressure, liquid water content of cloud (QC), precipitation, friction velocity, temperature, wind speed, and wind direction were considered. CMAQ v5.0.2 with HDDM was used to simulate $\mathrm{PM}_{2.5}$ concentrations and their first-order sensitivities to emissions, LBCs, and chemical reaction rates. The simulation period is April $10^{\text {th }}$ to $20^{\text {th }}, 2013$, when local sources and cross-boundary transport had similar impacts on $\mathrm{PM}_{2.5}$ formation in $\mathrm{PRD}^{30}$. Hourly measurements of $\mathrm{PM}_{2.5}$ concentrations from the Pearl River Delta Regional Air Quality Monitoring Network (PRDRAQM) were applied to evaluate and diagnose output uncertainties.

\section{RESULTS}

\subsection{Identification of important sensitivity inputs}

The sensitivities of $\mathrm{PM}_{2.5}$ concentrations in PRD to model inputs were analyzed (Figure 3 and Table S5). Because the target area of this case study is PRD, the model inputs considered for sensitivity analysis refer to those in domain 3 (D3) of the model system. Primary $\mathrm{PM}_{2.5}$ emission is the most sensitive emission input for $\mathrm{PM}_{2.5}$ simulations, with an RSC of $30.6 \%$, followed by $\mathrm{NH}_{3}(15.7 \%)$, $\mathrm{NOx}(10.4 \%), \mathrm{SO}_{2}(7.4 \%)$ and VOCs emissions (2.2\%). $\mathrm{NH}_{3}, \mathrm{NOx}$ and $\mathrm{SO}_{2}$ emissions are key precursors of aerosol formation, and thus also have noticeable impacts on SNA (sulfate, nitrate and ammonium) formation, as expected. In contrast, VOC emissions only have slight effects on $\mathrm{PM}_{2.5}$ simulations, despite being critical precursors of SOA, which typically accounts for $9 \sim 18 \%$ of $\mathrm{PM}_{2.5}$ concentrations in the PRD (Table S8). Further discussion of uncertainties in SOA is in the following uncertainty analysis. As expected, the simulated $\mathrm{PM}_{2.5}$ 
concentrations in D3 exhibited larger sensitivities to the $\mathrm{LBC} \mathrm{PM}_{2.5}$ and $\mathrm{O}_{3}$ concentrations. This is consistent with previous source apportionment studies in the PRD region, which indicated that a large portion of the $\mathrm{PM}_{2.5}$ concentrations attributed to $\mathrm{LBCs}^{31}$.

Wind speed and temperature are the two primary meteorological inputs that impact the $\mathrm{PM}_{2.5}$ simulations in this case study, with RSCs of $28.3 \%$ and $8.3 \%$, respectively, followed by relative humidity $(5.8 \%)$, wind direction (5.7\%), PBL (3.3\%), friction velocity (3.3\%) and precipitation $(2.6 \%)$. The wind speed and temperature are both negatively correlated with $\mathrm{PM}_{2.5}$ formations. Low wind speed enhances the accumulation of $\mathrm{PM}_{2.5}$ while high temperature promotes the volatility of nitrate and ammonium nitrate ${ }^{32}$. PBL height and precipitation do not have a significant effect on the simulated $\mathrm{PM}_{2.5}$ concentrations, likely stemming from the slight negative correlation between the PBL height and $\mathrm{PM}_{2.5}$ in $\mathrm{PRD}^{33}$ and the low precipitation during the simulation period.

The $\mathrm{NH}_{3}, \mathrm{NOx}, \mathrm{SO}_{2}$ and primary $\mathrm{PM}_{2.5}$ emissions, $\mathrm{PM}_{2.5}$ and $\mathrm{O}_{3}$ concentrations in LBCs, and temperature, wind speed and relative humidity were used for further uncertainty analysis. VOC emissions were also considered due to their relatively large uncertainties and our intention to analyze how their uncertainties impact SOA simulations. According to the IUPAC and JPL database, uncertainty ranges of most chemical reaction rates are within $20 \%{ }^{34,35}$, and thus chemical reaction rates were not considered owing to their comparatively low sensitivities and uncertainties (Table S5). The uncertainties (Table S6) in these selected inputs were quantified following the methods presented in S2 of SI. 


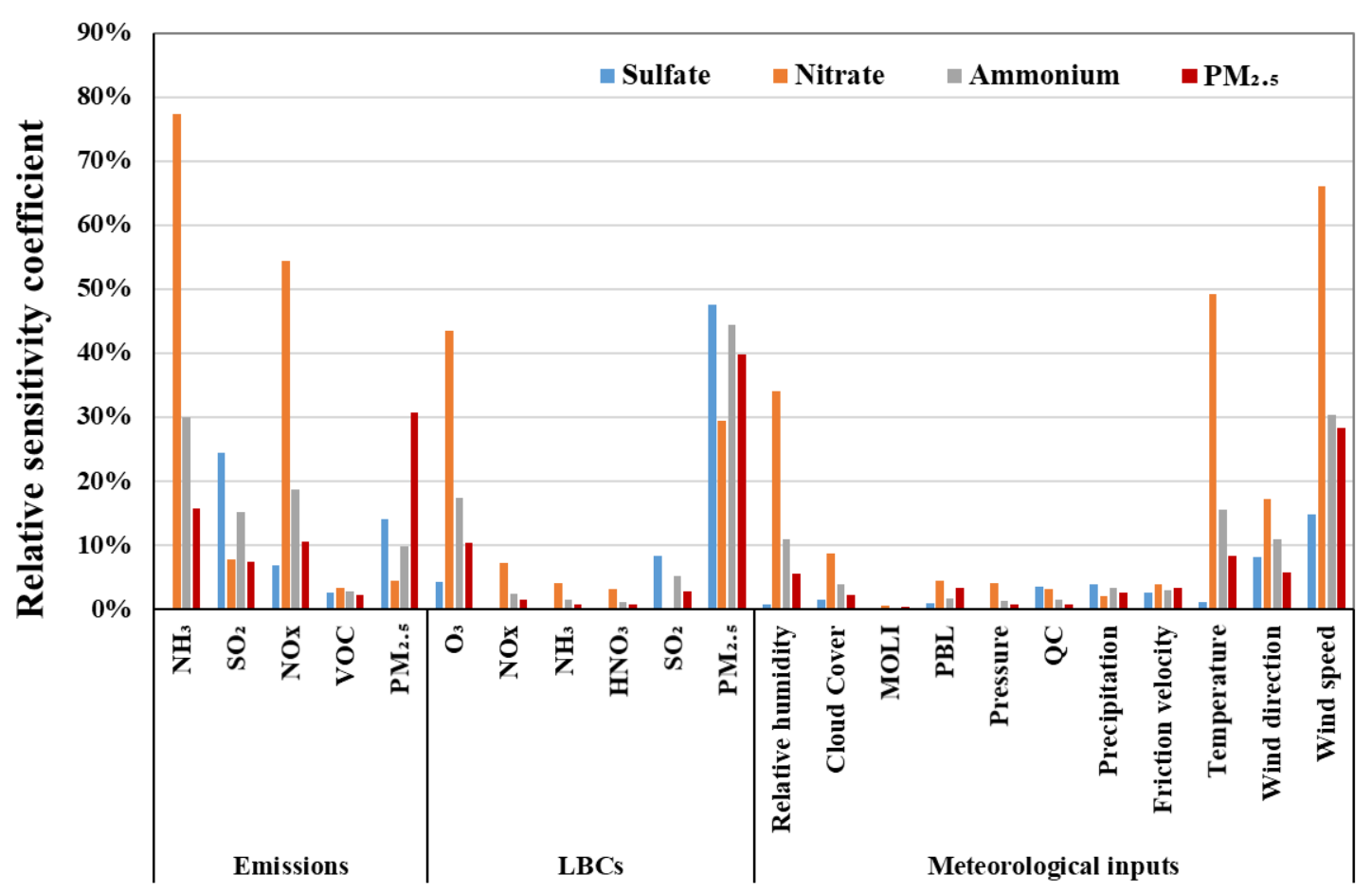

Figure 3. Relative sensitivity coefficients of $\mathrm{PM}_{2.5}$ and SNA concentrations averaged over all sites in PRDRAQM to emissions, LBCs, and meteorological fields. Their spatial patterns are shown in Figure S3S5.

\subsection{Evaluation of the HDDM-SRSM}

As introduced in Section 2.2, the HDDM-SRSM has the potential to improve efficiency while maintaining the accuracy of uncertainty propagation. Here, we evaluated the efficiency and accuracy in uncertainty propagation by comparing the second-order HDDM-SRSM, the thirdorder HDDM-SRSM, the third-order SRSM and the traditional MCM. These four approaches involved ten important model inputs, including five emission inputs, two LBC inputs and three meteorological inputs.

The second-order HDDM-SRSM is the most efficient of the four approaches tested; it only requires 28.6 hours to build a one-day PCE with ten inputs using a cluster applied in this study (Table S9), but it has large biases in uncertainty propagation (Figure 4). In comparison, the third-order HDDM-SRSM achieves a better balance between accuracy and efficiency. It requires 128.6 hours to build a one-day PCE, saving approximately $64 \%$ 
of the up-front computational cost compared with the third-order SRSM (357.5 hours). Also, the PDF of simulated $\mathrm{PM}_{2.5}$ concentrations estimated by the third-order HDDM-SRSM has a good agreement with that estimated by MCM, indicating that the third-order HDDM-SRSM can precisely propagate uncertainties. The third-order SRSM is also accurate, performing slightly better than the third-order HDDM-SRSM. Although the third-order SRSM had reduced computational cost compared to the MCM, which requires at least 1000 model runs and 812.6 hours for a precise propagation, it was still more computationally expensive compared to the third-order HDDM-SRSM. Therefore, the third-order HDDMSRSM is applied to the case study.

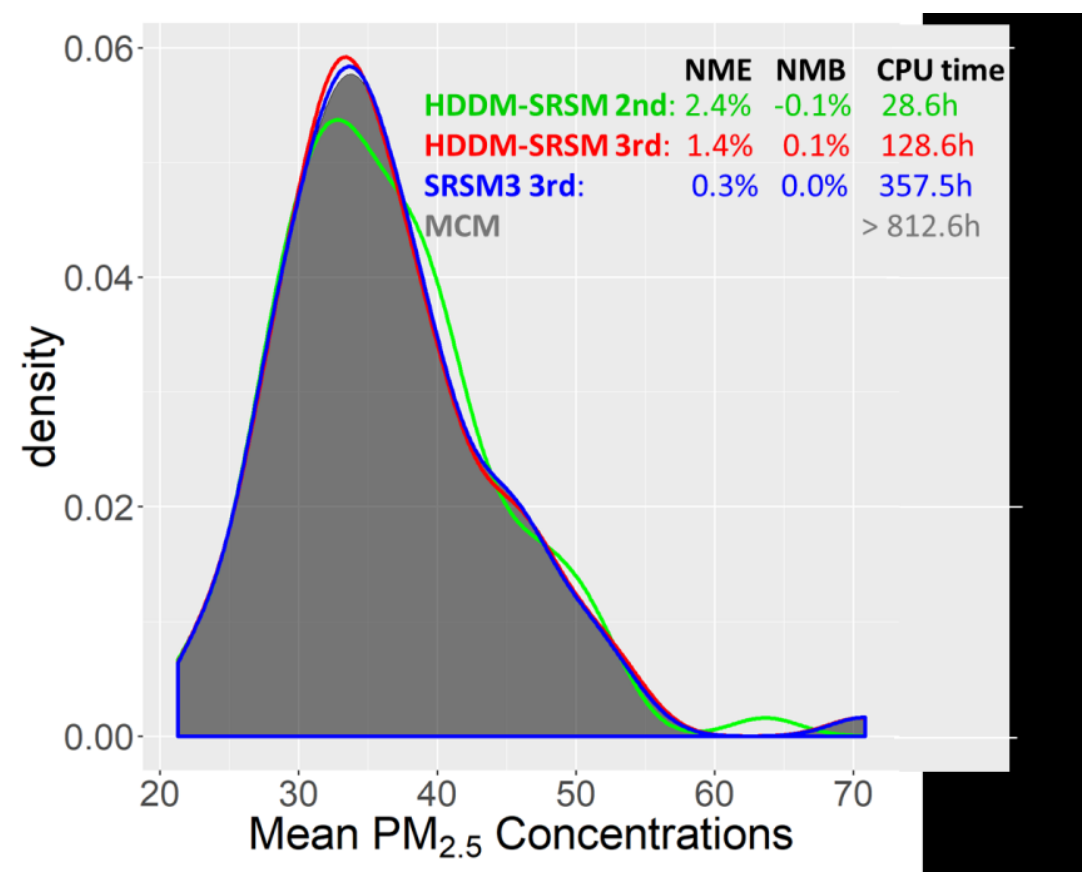

Figure 4. Comparison of the second-order HDDM-SRSM, the third-order HDDM-SRSM, the third-order SRSM and the MCM with respect to the accuracy and efficiency of uncertainty propagation. Accuracy was evaluated by comparing the PDFs, in which 200 random samples were used, of PCEs to those of MCM (the most accurate uncertainty propagation approach). Efficiency was evaluated by estimating the upfront computational costs required by RFM approaches using the Intel High-Performance Computing cluster with one node (CPU: $2 \times E 5-$ 2680V3). 


\subsection{Uncertainties in simulated $\mathbf{P M}_{2.5}$ concentrations}

Following the framework, uncertainties in the simulated $\mathrm{PM}_{2.5}$ concentrations were quantified (Figure 5). The evaluation of these uncertainties is shown in S8 of SI. Overall, the relative uncertainty, which is defined as the ratio of the $95 \%$ confidence interval (CI) to two times the median (S2 of SI for details) in simulated hourly $\mathrm{PM}_{2.5}$ concentrations at all sites associated with emissions, LBCs and meteorological inputs is $60.2 \%$ on average (the $95 \% \mathrm{CI}$ ranges from $-39.5 \%$ to $91.7 \%$ ) (Table S10). This uncertainty can cover approximately $80 \%$ of the hourly $\mathrm{PM}_{2.5}$ observations, indicating that uncertainties in emissions, LBCs, and meteorological inputs can account for most, but not all, of the $\mathrm{PM}_{2.5}$ simulation bias. $\mathrm{PM}_{2.5}$ simulation uncertainties associated with different uncertainty sources were also quantified. Because precursor emissions are mainly concentrated in the central PRD region (Figure S3), uncertainties in emission and meteorological inputs pose more impacts in the urban sites (e.g., GZPY) and downwind sites (e.g., JMDH). In contrast, the effects of uncertainties in LBCs are larger at upwind sites (e.g., CHTH) that are located near domain boundaries.

There are higher uncertainties in simulated SNA species $(186.3 \%$ of relative uncertainty for nitrate, $81.3 \%$ for ammonium and $61.7 \%$ for sulfate), than in simulated $\mathrm{PM}_{2.5}(60.2 \%)$. In particular, the simulated nitrate has the largest uncertainty and is the most susceptible to emissions, LBCs, and meteorological inputs. This may be contributing to the poor performance of nitrate simulation in $\mathrm{CTMs}^{3}$. Despite the high SNA uncertainties, the uncertainty ranges estimated in this case study still can cover $78 \%, 84 \%$ and $89 \%$ of observed sulfate, nitrate and ammonium, respectively (Figure 5 d-f). Furthermore, the uncertainty means of both $\mathrm{PM}_{2.5}$ mass concentrations and SNA specie concentrations are more consistent with observations, particularly in the period of April $14-15$, 
2013, when the base estimate largely underestimates $\mathrm{PM}_{2.5}$ concentrations. This indicates that uncertainty analysis can improve the model performance in $\mathrm{PM}_{2.5}$ simulations, not only in mass concentrations but also in SNA species.

The average of uncertainty in $\mathrm{PM}_{2.5}$ mass concentration improves the model performance; however, it is still systematically underestimated when evaluated with observations (Figure 5 and Figure S6). This likely arises from SOA underprediction. Based upon the uncertainty analysis, there is an overestimate of the uncertainty mean of SNA with the NMB of $1.5 \%$ (Figure $\mathrm{S} 8$ ). However, the NMB of $\mathrm{PM}_{2.5}$ mass is $-15.2 \%$. Also, although the uncertainty of VOCs emissions estimated in this study ranges from $-50 \%$ to $+100 \%$, the uncertainty of simulated SOA concentrations is approximately $4.2-5.2 \mu \mathrm{g} / \mathrm{m}^{3}$, which is significantly lower than the average of the observed SOA concentrations $\left(7.5-14.2 \mu \mathrm{g} / \mathrm{m}^{3}\right)$ estimated from field campaigns (Table S8). These two facts imply that the significant SOA underestimation can be attributed to the limitation of the two-product method applied in CMAQv5.0.2 to simulate SOA. Indeed, this finding is consistent with previous studies that revealed the systematic SOA underestimation using the two-product method ${ }^{36,37}$. The quantitative uncertainty analysis is shown to be competent for CTMs diagnosis. 

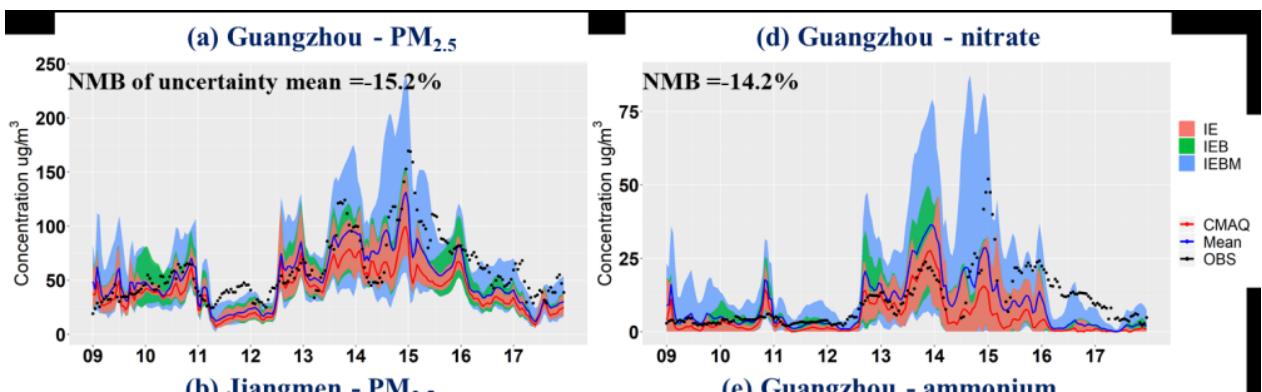

(e) Guangzhou - ammonium
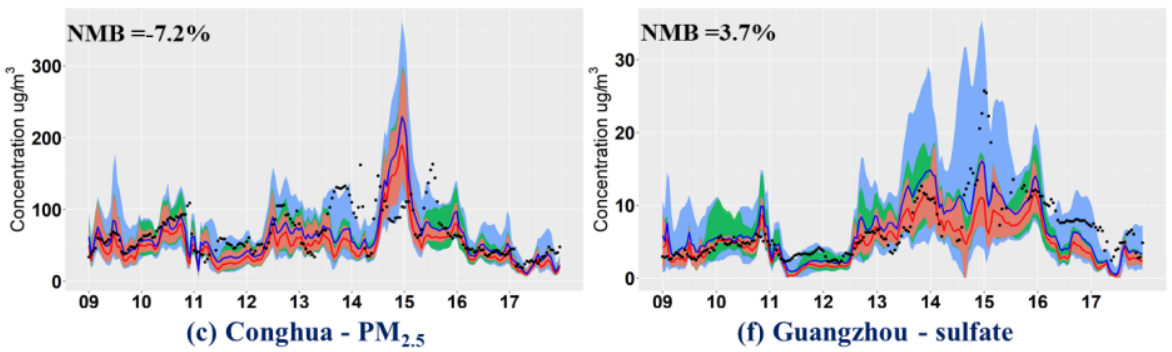

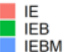
(c) Conghua - $\mathrm{PM}_{2.5}$
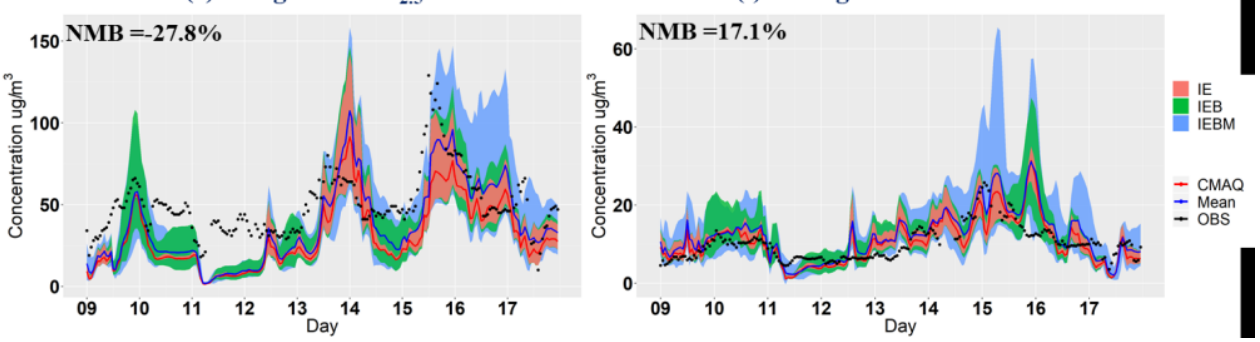

Figure 5. Time series of hourly $\mathrm{PM}_{2.5}$ concentrations at (a) Guangzhou Panyu (GZPY), (b) Jiangmen (JMDH) and (c) Conghua (CHTH). At Guangzhou Panyu site, time series of (d) nitrate, (e) ammonium and (f) concentrations at the base case. The blue shaded area is the $95 \% \mathrm{CI}$ of $\mathrm{PM}_{2.5}$ concentrations associated with emissions, LBCs, and meteorology (IEBM). The green shaded area is the uncertainty range associated with emissions and LBCs (IEB). The red shaded area is the uncertainty range associated with emissions. The black points are observed $\mathrm{PM}_{2.5}$ concentrations. Guangzhou, Jiangmen, and Conghua are located in the urban, rural and downwind areas of the PRD region.

uncertainty sources for $\mathrm{PM}_{2.5}$ simulations, which together account for $81.4 \%$ of the total uncertainty in simulated $\mathrm{PM}_{2.5}$ concentrations (Figure 6). The

374 primary $\mathrm{PM}_{2.5}$ emissions generally have high uncertainty in China due to

375 the limited measurements of local emission factors and a dearth of detailed activity data, particularly for fugitive dust, one of the largest contributors 
377 to primary $\mathrm{PM}_{2.5}$ emissions ${ }^{38}$. The key uncertainty sources identified in this

378 case study have previously been uncovered by Huang et al. $^{39}$. In that study, 379 the bias in LBCs for the PRD domain (D3) was reduced using an optimized 380 data fusion method that combines model output and observations. The 381 evaluation showed that reducing uncertainty in LBCs improves $\mathrm{PM}_{2.5}$ 382 simulations, with fractional bias decreased by $3-15 \%$. This indicates that 383 the enhancement of key uncertainty sources can indeed improve model performances.

385 The key uncertainty sources for SNA simulations are different from 386 those of $\mathrm{PM}_{2.5}$ simulations. There is lower uncertainty contribution from 387 primary $\mathrm{PM}_{2.5}$ emissions, which is reasonable considering that primary $388 \mathrm{PM}_{2.5}$ emissions contain fewer SNA species. Temperature and $\mathrm{NH}_{3}$ 389 emissions are the two leading key uncertainty sources for nitrate 390 simulations. This is as expected because $\mathrm{NH}_{3}$ emissions are a critical 391 precursor of ammonium nitrate aerosol formation, and the formation is 392 strongly dependent on temperature ${ }^{32}$. Moreover, $\mathrm{NH}_{3}$ emissions have high 393 uncertainties in China due to the limited activity data and less 394 representative emission factors ${ }^{38}$. Thereby, reducing the uncertainties in $395 \mathrm{NH}_{3}$ emissions and temperature could improve SNA simulations. 


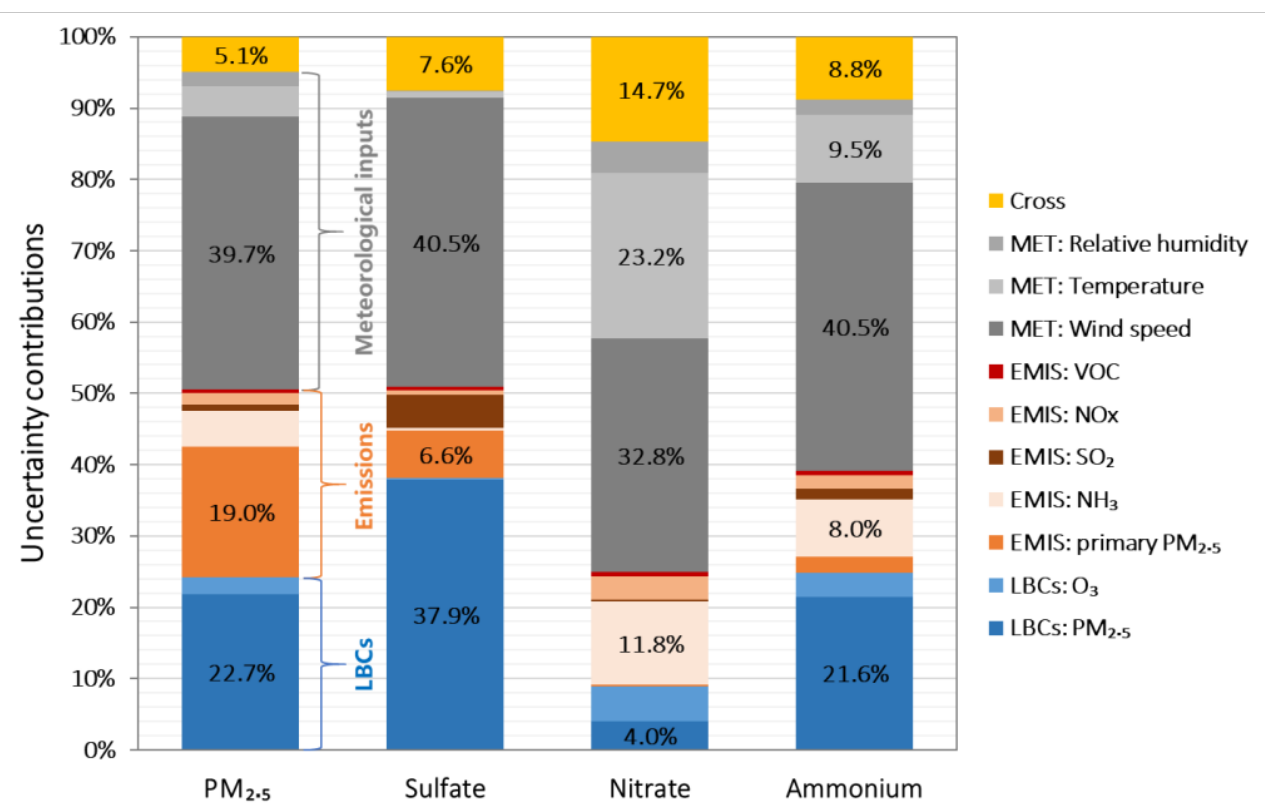

Figure 6. Contributions of uncertainty inputs to uncertainties in simulated $\mathrm{PM}_{2.5}$ concentrations averaged at all sites in PRDRAQM. MET denotes meteorological fields, EMIS denotes emissions in D3 and LBCs denotes lateral boundary conditions.

\section{DISCUSSION}

Quantitative uncertainty analysis is an essential approach to identify key uncertainty sources for model diagnosis. However, this approach has only been applied in specific cases in CTMs because current uncertainty analysis approaches (e.g., RFM-DDM, SRSM, and MCM) suffer from either inaccuracy or inefficiency or both in certain cases. In this study, we proposed a methodological framework for the uncertainty analysis of CTMs, featuring the use of sensitivity analysis to filter out unimportant model inputs and the use of a new coupling HDDM-SRSM approach to improve the efficiency and accuracy of uncertainty propagation. The case study of PRD region shows that the framework is feasible in efficiently identifying key uncertainty sources and accurately propagating uncertainties of model inputs through CTM models while reducing computational resources (64\% saving compared to the SRSM and $90 \%$ 
saving compared to the MCM). The uncertainty analysis of one-day model simulation takes 128.6 hours on one node for the case study, but the computational time can be reduced to a few hours with the use of multiple nodes (Intel CPU with 50 nodes, see Table S9), making it feasible to conduct operational probabilistic air quality forecasting. In addition, this framework can be extended to other widely used CTM models.

The case study demonstrates the uncertainty analysis is effective in model diagnosis and guiding model improvements. For example, the preliminary uncertainty analysis showed a systematic underestimate from the two-product method applied to describe SOA formation in CMAQv5.0.2. Using the volatility basis set (VBS) ${ }^{37}$, a new SOA module, the simulated SOA concentrations increased by $16 \%$ which was much closer to SOA observations (Figure S11). It demonstrates the critical role of the uncertainty analysis in diagnosing and improving CTM models. With further uncertainty analysis, it is possible that other systematic biases can be diagnosed. Also, in the case study, we identified primary $\mathrm{PM}_{2.5}$ emissions, $\mathrm{PM}_{2.5}$ concentration in LBCs and wind speed as key uncertainty sources. Our work validated the enhancement of these key uncertainty sources could indeed improve model performance ${ }^{39}$. However, it must be pointed out that key uncertainty sources might vary from case to case, depending on the geographic domains, simulation periods, emissions, weather conditions, and chemical processes. For example, LBCs becomes the largest uncertainty source $(55.2 \%)$ of $\mathrm{PM}_{2.5}$ simulations in December when the $\mathrm{PM}_{2.5}$ formation in the PRD is affected mainly by cross-boundary transport. However, it only contributes $22.7 \%$ the uncertainty in $\mathrm{PM}_{2.5}$ simulations in April (Figure S9). In this case study, PBL is a minor uncertainty source within the ten-day simulation period, but PBL might emerge as a key uncertainty source if the simulation period was extended to one-year span. This also indicates that model improvements should 
focus on different model inputs in different simulation cases.

Apart from diagnosing CTM models, the uncertainty analysis can be applied to improve the model performance and the reliability of air quality forecasting by using the uncertainty mean and tailoring the uncertainty to get the probabilistic information. As shown in the case study, the uncertainty mean have a better agreement with observations than deterministic estimates. Apart from the uncertainty mean, the peak of uncertainty distribution and the uncertainty median are also better predictors (Table S7). Additionally, the probabilistic information can make the air quality forecasting more reliable. Here, we used a case example (Figure S10) to illustrate this, which was calibrated by the reliability diagram (Figure S7). The simulated daily $\mathrm{PM}_{2.5}$ based on deterministic simulation on the day of April 13, 2013 was $74 \mu \mathrm{g} / \mathrm{m}^{3}$, which did not exceed the national grade II standard $\left(75 \mu \mathrm{g} / \mathrm{m}^{3}\right)$. Thereby, air quality was forecasted to be "good" according to the Chinese Air Quality Forecast Regulation. However, the observed $\mathrm{PM}_{2.5}$ concentration was $95 \mu \mathrm{g} / \mathrm{m}^{3}$ on that day, which was at the "slightly polluted" level. If we used the probabilistic information, the likelihood of the "good" level was only $35 \%$, while the likelihood of the "slightly polluted" was $65 \%$, and the uncertainty mean was $90 \mu \mathrm{g} / \mathrm{m}^{3}$, giving us sufficient confidence to forecast the air quality as "slightly polluted" level, which was more consistent with the observation. The framework has the ability to add quantitative probabilistic information to forecasts, which is feasible regarding the time requirement. Compared with traditional probabilistic air quality forecasting that heavily relies on ensemble simulation, the framework is able to consider parametric uncertainties and identify key uncertainty sources to further improve forecasting performance ${ }^{7}$. Coupled with the ensemble method, structural uncertainties can also be addressed under the framework ${ }^{2}$.

Although the high-order HDDM-SRSM showed high accuracy in 
propagation, it does not mean that the accuracy of PCEs developed by HDDM-SRSM is held over all CTM simulations, partly due to the overfitting issue that typically occurs in cases with many unknown coefficients but fewer collocation points, such as 286 unknown coefficients and 81 collocation points in this study (Figure S1). When a PCE is overfitting, it performances well in interpolation but generally has biases in extrapolation. In HDDM-SRSM, all collocation points for estimating unknown coefficients almost fall in the region of high probability of inputs. It means that the best performance of HDDM-SRSM is restricted to simulations within the range of input uncertainties $(95 \% \mathrm{CI})$. Beyond the range, the PCE is not adequately represented. Therefore, if input uncertainties have substantial changes, the PCE must be rebuilt according to the new input uncertainties to secure accurate uncertainty propagations.

\section{- ASSOCIATED CONTENT}

Supporting Information: Information on sensitivity analysis (S1, S7), uncertainties in model inputs (S2), uncertainty evaluation (S3, S8), uncertainty attribution (S4), details of HDDM-SRSM (S5), model setup (S6), and other figures and tables for case study (S9)

\section{- AUTHOR INFORMATION}

\section{Corresponding Author}

* Junyu Zheng. Phone: +86-20-37336635; fax: +86-20-37336635; e-mail: zheng.junyu@gmail.com;

\section{- ACKNOWLEDGMENT}

This work was supported by National Distinguished Young Scholar Science Fund of the National Natural Science Foundation of China (No. 41325020). 
1. Isukapalli, S. S.; Roy, a.; Georgopoulos, P. G., Stochastic response surface methods (SRSMs) for uncertainty propagation: Application to environmental and biological systems. Risk.Anal. 1998, 18, 351-363.

2. Pinder, R. W.; Gilliam, R. C.; Appel, K. W.; Napelenok, S. L.; Foley, K. M.; Gilliland, A. B., Efficient Probabilistic Estimates of Surface Ozone Concentration Using an Ensemble of Model Configurations and Direct Sensitivity Calculations. Environ. Sci. Technol. 2009, 43, 2388-2393.

3. Simon, H.; Baker, K. R.; Phillips, S., Compilation and interpretation of photochemical model performance statistics published between 2006 and 2012. Atmos. Environ. 2012, 61, 124-139.

4. Fine, J.; Vuilleumier, L.; Reynolds, S.; Roth, P.; Brown, N., Evaluating uncertainties in regional photochemicalair quality modeling. Annu. Rev. Environ. Resour. 2003, 28, 59-106. 5. Russell, A.; Dennis, R., NARSTO critical review of photochemical models and modeling. Atmos. Environ. 2000, 34, 2283-2324. 6. Tian, D.; Cohan, D. S.; Napelenok, S.; Bergin, M.; Hu, Y.; Chang, M.; Russell, A. G., Uncertainty Analysis of Ozone Formation and Response to Emission Controls Using HigherOrder Sensitivities. J. Air Waste Manage. Assoc. 2010, 60, 797-804.

7. Huang, Z.; Hu, Y.; Zheng, J.; Yuan, Z.; Russell, A. G.; Ou, J.; Zhong, Z., A New Combined Stepwise-Based High-Order Decoupled Direct and Reduced-Form Method to Improve Uncertainty Analysis in PM 2.5 Simulations. Environ. Sci. Technol. 2017, 51, 38523859.

8. Zhang, W.; Trail, M. A.; Hu, Y.; Nenes, A.; Russell, A. G., Use of high-order sensitivity analysis and reduced-form modeling to quantify uncertainty in particulate matter simulations in the presence of uncertain emissions rates: A case study in Houston. Atmos. Environ. 2015, $122,103-113$.

9. World Climate Research Programme. WCRP Strategic Plan 2019-2029; WCRP Publication No. 1/2019; World Meteorological Organization: Geneva, 2018. 10. Dabberdt, W. F.; Miller, E., Uncertainty, ensembles and air quality dispersion modeling: applications and challenges. Atmos. Environ. 2000, 34, 4667-4673.

11. Beekmann, M., Monte Carlo uncertainty analysis of a regional-scale transport chemistry model constrained by measurements from the Atmospheric Pollution Over the Paris Area (ESQUIF) campaign. J. Geophys. Res. 2003, 108, 8559.

\section{Moore, G. E.; Londergan, R. J., Sampled Monte Carlo uncertainty analysis for} photochemical grid models. Atmos. Environ. 2001, 35, 4863-4876.

13. Bergin, M. S.; Noblet, G. S.; Petrini, K.; Dhieux, J. R.; Milford, J. B.; Harley, R. A., 
Formal Uncertainty Analysis of a Lagrangian Photochemical Air Pollution Model. Environ.

541 Sci. Technol. 1999, 33, 1116-1126.

542 14. Tatang, M. A.; Pan, W.; Prinn, R. G.; McRae, G. J., An efficient method for parametric

543 uncertainty analysis of numerical geophysical models. J. Geophys. Res.: Atmos. 1997, 102,

$544 \quad 21925-21932$.

545 15. Thackray, C. P.; Friedman, C. L.; Zhang, Y. X.; Selin, N. E., Quantitative Assessment of

546 Parametric Uncertainty in Northern Hemisphere PAH Concentrations. Environ. Sci. Technol.

$547 \quad 2015,49(15), 9185-9193$.

548 16. Lucas, D. D.; Prinn, R. G., Parametric sensitivity and uncertainty analysis of

549 dimethylsulfide oxidation in the clear-sky remote marine boundary layer. Atmos. Chem. Phys.

$5502005,5,1505-1525$.

551 17. Cheng, H. Y.; Sandu, A., Uncertainty quantification and apportionment in air quality

552 models using the polynomial chaos method. Environ. Model. Softw. 2009, 24 (8), 917-925.

553 18. Foley, K. M.; Reich, B. J.; Napelenok, S. L., Bayesian analysis of a reduced-form air

554 quality model. Environ. Sci. Technol. 2012, 46, 7604-11.

555 19. Foley, K. M.; Napelenok, S. L.; Jang, C.; Phillips, S.; Hubbell, B. J.; Fulcher, C. M., Two

556 reduced form air quality modeling techniques for rapidly calculating pollutant mitigation

557 potential across many sources, locations and precursor emission types. Atmos. Environ. 2014,

$55898,283-289$.

559 20. Isukapalli, S. S.; Roy, A.; Georgopoulos, P. G., Efficient sensitivity/uncertainty analysis

560 using the combined stochastic response surface method and automated differentiation:

561 application to environmental and biological systems. Risk.Anal. 2000, 20, 591-602.

562 21. Cacuci, D. G., Sensitivity and Uncertainty Analysis: Theory, Volume I; CRC Press, 2003.

563 22. Fahey, K. M.; Carlton, A. G.; Pye, H. O. T.; Baek, J.; Hutzell, W. T.; Stanier, C. O.;

564 Baker, K. R.; Wyat Appel, K.; Jaoui, M.; Offenberg, J. H., A framework for expanding

565 aqueous chemistry in the Community Multiscale Air Quality (CMAQ) model version 5.1.

566 Geosci. Model Dev. 2017, 10, 1587-1605.

567 23. Loucks, D. P.; Beek, E. v.; Stedinger, J. R.; Dijkman, J. P. M.; Villars, M. T., Water

568 Resources Planning and Management: An Overview; United Nations Educational, Scientific

569 and Cultural Organization: Paris, 2005.

570 24. Hamby, D. M., A review of techniques for parameter sensitivity analysis of

571 environmental models. Environ. Monit. Assess. 1994, 32, 135-54.

572 25. Zhang, W.; Capps, S. L.; Hu, Y.; Nenes, A.; Napelenok, S. L.; Russell, a. G.,

573 Development of the high-order decoupled direct method in three dimensions for particulate

574 matter: Enabling advanced sensitivity analysis in air quality models. Geosci. Model Dev.

$575 \quad 2012,5,355-368$.

576 26. Casati, B.; Wilson, L. J.; Stephenson, D. B.; Nurmi, P.; Ghelli, A.; Pocernich, M.; 
577 Damrath, U.; Ebert, E. E.; Brown, B. G.; Mason, S. Forecast Verification: Current Status and 578 Future Directions. Meteorol. Appl. 2008, 15 (1), 3-18.

579 27. Dennis, R.; Fox, T.; Fuentes, M.; Gilliland, A.; Hanna, S.; Hogrefe, C.; Irwin, J.; Rao, S.

580 T.; Scheffe, R.; Schere, K.; et al. A Framework for Evaluating Regional-Scale Numerical

581 Photochemical Modeling Systems. Environ. Fluid Mech. 2010, 10 (4), 471-489.

582 28. Gneiting T, B. F.; AE, R. Probabilistic Forecasts, Calibration and Sharpness. J. R. Stat.

583 Soc. Ser. B (Statistical Methodology). 2007, 69, 243-268.

584 29. Hamill, T. M. Reliability Diagrams for Multicategory Probabilistic Forecasts. Weather

585 Forecast. 1997, 12 (4), 736-741.

586 30. Wu, D.; Fung, J. C. H.; Yao, T.; Lau, A. K. H., A study of control policy in the Pearl

587 River Delta region by using the particulate matter source apportionment method. Atmos.

588 Environ. 2013, 76, 147-161.

589 31. Yin, X.; Huang, Z.; Zheng, J.; Yuan, Z.; Zhu, W.; Huang, X.; Chen, D., Source

590 contributions to PM2.5 in Guangdong province, China by numerical modeling: Results and

591 implications. Atmos. Res. 2017, 186, 63-71.

592 32. Makar, P. A.; Wiebe, H. A.; Staebler, R. M.; Li, S. M.; Anlauf, K., Measurement and 593 modeling of particle nitrate formation. J. Geophys. Res. 1998, 103, 13095-13110.

594 33. Su, T.; Li, Z.; Kahn, R. Relationships between the Planetary Boundary Layer Height and

595 Surface Pollutants Derived from Lidar Observations over China: Regional Pattern and

596 Influencing Factors. Atmos. Chem. Phys. 2018, 18 (21), 15921-15935.

597 34. Sander, S. P.; Friedl, R. R.; Barker, J. R.; Golden, D. M.; Kurylo, M. J.; Wine, P. H.;

598 Abbatt, J. P. D.; Burkholder, J. B.; Kolb, C. E.; Moortgat, G. K.; et al. Table of Contents

599 Chemical Kinetics and Photochemical Data for Use in Atmospheric Studies: Evaluation

600 Number 17; JPL Publication 10-6; California Institute of Technology: California, 2011.

601 35. Atkinson, R.; Baulch, D. L.; Cox, R. a.; Crowley, J. N.; Hampson, R. F.; Hynes, R. G.;

602 Jenkin, M. E.; Rossi, M. J.; Troe, J., Evaluated kinetic and photochemical data for

603 atmospheric chemistry: Volume III - gas phase reactions of inorganic halogens. Atmos.

604 Chem. Phys. 2007, 7, 981-1191.

605 36. Guo, X.-S.; Situ, S.-P.; Wang, X.-M.; Ding, X.; Wang, X.-M.; Yan, C.-Q.; Li, X.-Y.;

606 Zheng, M. Numerical Modeling Analysis of Secondary Organic Aerosol (SOA) Combined

607 with the Ground-Based Measurements in the Pearl River Delta Region. Environ. Sci. 2014, 35

608 (5), 1654-1661.

609 37. Li, J.; Zhang, M.; Wu, F.; Sun, Y.; Tang, G., Assessment of the impacts of aromatic VOC

610 emissions and yields of SOA on SOA concentrations with the air quality model RAMS-

611 CMAQ. Atmos. Environ. 2017, 158, 105-115.

612 38. Zhong, Z.; Zheng, J.; Zhu, M.; Huang, Z.; Zhang, Z.; Jia, G.; Wang, X.; Bian, Y.; Wang,

613 Y.; Li, N., Recent developments of anthropogenic air pollutant emission inventories in 
614 Guangdong province, China. Sci. Total Environ. 2018, 627, 1080-1092.

615 39. Huang, Z.; Hu, Y.; Zheng, J.; Zhai, X.; Huang, R., An optimized data fusion method and 616 its application to improve lateral boundary conditions in winter for Pearl River Delta regional $617 \quad \mathrm{PM}_{2.5}$ modeling, China. Atmos. Environ. 2018, 180, 59-68. 